\title{
Approximate BER for OFDM Systems Impaired by a Gain Mismatch of a TI-ADC Realization
}

\author{
Vo-Trung-Dung Huynh, Nele Noels, Heidi Steendam \\ Department of Telecommunications and Information Processing, Ghent University \\ \{votrungdung.huynh, nele.noels, heidi.steendam\}@ugent.be
}

\begin{abstract}
Time-interleaved analog-to-digital converters (TIADCs) have recently been suggested for multi-Gigabit systems because of their high sampling rate. In practice, a major bottleneck of using a TI-ADC is mismatch occurring between individual sub-ADCs. In this paper, we derive an approximate bit error rate (BER) expression for high-speed pulse amplitude modulated (PAM) and quadrature amplitude modulated (QAM) orthogonal frequency division multiplexing (OFDM) receivers employing a TI-ADC with gain mismatch between the sub-ADCs. We assume an additive white Gaussian noise (AWGN) channel and binary reflected Gray code mapping. The numerical results show that the derived BER expression is in excellent agreement with Monte-Carlo simulation results if the number $L$ of subADCs is sufficiently large, but becomes less accurate for smaller values of $L$.
\end{abstract}

Index Terms-BER, OFDM, TI-ADC, gain mismatch, AWGN channel.

\section{INTRODUCTION}

Orthogonal frequency division multiplexing (OFDM) is widely used for broadband wired and wireless communication systems because of its high spectral efficiency and tolerance against inter-symbol interference (ISI) caused by a dispersive channel [1]. Recently, it has been suggested for fibre-optic communication systems, in which the data rate increases to $100 \mathrm{~Gb} / \mathrm{s}$ and beyond [2]. In such high-speed OFDM systems, the analog-to-digital converter (ADC), which is placed in front of the baseband digital signal processing (DSP) unit, is required to operate at an extremely high sampling rate. The operating sample rate of a regular ADC is limited by the physical constraints of the employed technology [3]. A timeinterleaved (TI) architecture of $L$ identical ADCs allows to achieve a sampling rate $\frac{1}{T_{s}}$, although each individual ADC is actually sampling at a lower rate of $\frac{1}{L T_{s}}$. As illustrated in the model shown in Fig. 1, the $L$ sub-ADCs sample the analog input signal in a periodic manner, with as time spacing the ideal sampling time $T_{s}$, and produce a combined digital output signal. A drawback of TI-ADCs is that mismatches between the sub-ADCs can cause a system performance degradation [4]. A particular type of mismatch in a TI-ADC is gain mismatch which originates from differences in the capacitors' gain as well as in the finite opamps' gain among parallel subADCs [5]. The impact of the gain mismatch on the system performance has recently been studied for both single-carrier [6-8] and multi-carrier [4,9] systems. However, to the best of our knowledge, a closed-form bit error rate (BER) expression for OFDM systems employing a TI-ADC with gain mismatch

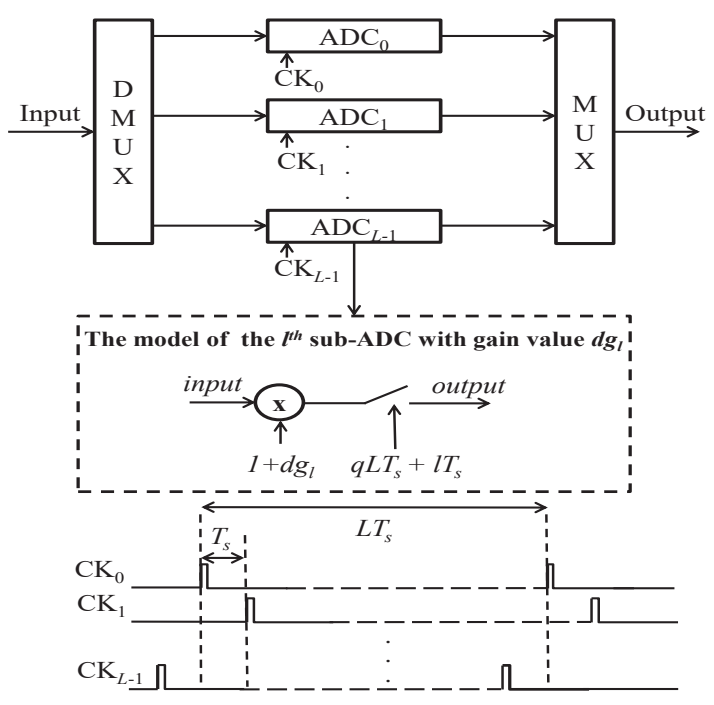

Fig. 1. Block diagram of a TI-ADC and a model of the $l^{\text {th }}$ sub-ADC with gain value $d g_{l}$.

has not been derived yet. In this paper, we analytically derive such a BER expression based on a Gaussian approximation of the interference. We restrict our attention to an additive white Gaussian noise (AWGN) channel in order to be able to isolate the effect of the gain mismatch. Further, we assume quadrature amplitude modulation (QAM) and bit-to-symbol mapping based on the binary reflected Gray code (BRGC) [10]. The accuracy of the derived BER expression is assessed by comparing the numerical evaluations with Monte-Carlo BER simulations. A good agreement between the theoretical and the empirical results is observed provided that the number $L$ of sub-ADCs is sufficiently large.

The paper is organized as follows. Section II describes the system model. The BER expression for a rectangular QAM constellation is derived in Section III. The BER expressions for square QAM and pulse amplitude modulation (PAM) constellations are special cases thereof. Section IV provides numerical results to validate the correctness of the derived BER expression. Section V concludes the paper with some final remarks.

\section{System Model}

Fig. 2 illustrates the block diagram of the considered OFDM system. The receiver is assumed to employ a TI- 


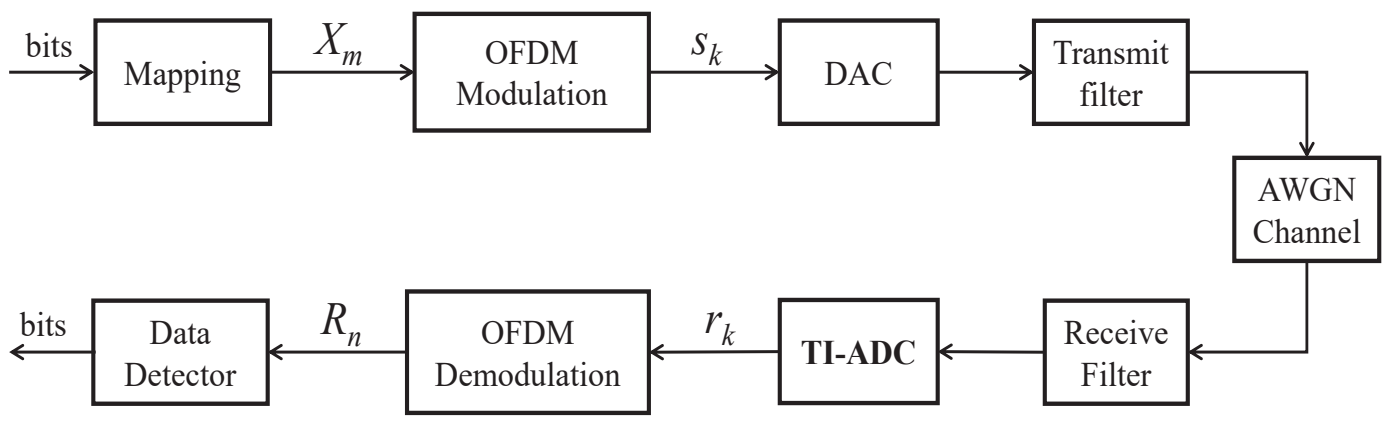

Fig. 2. Block diagram of an OFDM system with a TI-ADC at the receiver.

ADC in which the different sub-ADCs experience different gain mismatch values. The input/output behavior of the $l^{\text {th }}$ sub-ADC with gain mismatch value $d g_{l}$ is illustrated in Fig. 1. To simplify the notations, we further consider the transmission of a single OFDM block $\mathbf{X}$. The vector $\mathbf{X}$ consists of an even number $N$ of complex-valued symbols, i.e., $\mathbf{X}=\left(X_{-\frac{N}{2}}, X_{-\frac{N}{2}+1}, \ldots, X_{\frac{N}{2}-1}\right)^{T}$, which are taken from a unit-energy $M_{I} \times M_{Q}$ rectangular QAM constellation. Each constellation symbol is formed by mapping a sequence of $\left(m_{I}+m_{Q}\right)$ information bits $\left(m_{I}=\log _{2} M_{I}, m_{Q}=\log _{2} M_{Q}\right)$ onto the in-phase (I) and the quadrature $(\mathrm{Q})$ components of a rectangular QAM constellation using the 2-dimensional $m_{I^{-}}$ by- $m_{Q}$ bit BRGC mapping rule [10]. A size- $N$ inverse discrete Fourier transform (IDFT) unit converts the data symbols $\mathbf{X}$ to the samples $s_{k}$, given by:

$s_{k}=\frac{1}{\sqrt{N}} \sum_{m=-\frac{N}{2}}^{\frac{N}{2}-1} X_{m} e^{j 2 \pi \frac{m k}{N}}, k=-\frac{N}{2},-\frac{N}{2}+1, \ldots, \frac{N}{2}-1$.

Before transmission over an AWGN channel, the samples $s_{k}$ from (1) are converted to a continuous-time signal by a digitalto-analog converter (DAC) and a transmit filter is applied to eliminate the transmitted signal's images created by the DAC.

At the receiver, we assume perfect timing synchronization and matched filtering. After passing through the receive filter, a TI-ADC consisting of $L$ parallel sub-ADCs samples the received waveform at Nyquist rate $\frac{1}{T_{s}}$. The TI-ADC is assumed to have a sufficiently high resolution, so the quantization noise can be neglected [11]. Further, since in practice, the gain values $d g_{l}$ of the individual sub-ADCs vary only slowly in time [9], they will be modelled as constants over the duration of an OFDM symbol period. Using the model from [8], the output of the TI-ADC with gain mismatch can be written as:

$$
\begin{aligned}
r_{k}=\sum_{l=0}^{L-1} \sum_{q=-\infty}^{+\infty}\left(1+d g_{l}\right)\left(\sqrt{E_{s}} s_{k}+w_{k}\right) \cdot \delta_{k-q L-l}, & \\
& k=-\frac{N}{2},-\frac{N}{2}+1, \ldots, \frac{N}{2}-1,
\end{aligned}
$$

where $r_{k}$ denotes the $k^{t h}$ received sample, $E_{s}$ is the transmitted symbol energy, $\delta_{k}$ denotes the discrete dirac function, $s_{k}$ is defined in (1), and $\left\{w_{k}\right\}$ are independent and identically distributed (i.i.d.) AWGN noise samples with zero mean and variance $\sigma_{w}^{2}=\frac{N_{0}}{2}$ per I/Q dimension. The samples $r_{k}$ are applied to a size- $N$ discrete Fourier transform (DFT) unit. The output of the DFT unit is a vector $\mathbf{R}=\left(R_{-\frac{N}{2}}, R_{-\frac{N}{2}+1}, \ldots, R_{\frac{N}{2}-1}\right)$ with

$$
\begin{aligned}
R_{n} & =\frac{1}{\sqrt{N}} \sum_{k=-\frac{N}{2}}^{\frac{N}{2}-1} r_{k} e^{-j 2 \pi \frac{k n}{N}} \\
& =\sqrt{E_{s}}\left(1+D G_{0}\right) X_{n}+\sqrt{E_{s}} \sum_{i=-\frac{L}{2}, i \neq 0}^{\frac{L}{2}-1} D G_{i} X_{n-i \frac{N}{L}} \\
& +\sum_{i=-\frac{L}{2}}^{\frac{L}{2}-1} D G_{i} W_{n-i \frac{N}{L}}+W_{n}, \\
& n=-\frac{N}{2},-\frac{N}{2}+1, \ldots, \frac{N}{2}-1,
\end{aligned}
$$

where $X_{n}$ are the transmitted symbols, $W_{n}$ are statistically independent Gaussian random variables with zero mean and variance $\sigma_{w}^{2}=\frac{N_{0}}{2}$, and $D G_{i}$ is a function of the gain mismatch values $d g_{l}$ of the $L$ parallel sub-ADCs:

$$
D G_{i}=\frac{1}{L} \sum_{l=0}^{L-1} d g_{l} e^{-j 2 \pi \frac{i l}{L}}
$$

In (3), it is assumed for notational convenience that the number $L$ of sub-ADCs is a power of 2 , and the ratio $\frac{N}{L}$ between the DFT size and the number of sub-ADCs is an integer value. However, extension to non-integer values of $\frac{N}{L}$ is straightforward. The quantities $R_{n}$ in (3) are used to perform bit sequence detection by mapping them to the nearest constellation point and applying the inverse mapping rule.

\section{BER DERIVATION}

The overall BER is computed as the average BER of all $N_{d}$ data-modulated sub-carriers:

$$
B E R=\frac{1}{N_{d}} \sum_{n \in I_{d}} B E R_{n},
$$

where $B E R_{n}$ denotes the BER for the $n^{t h}$ sub-carrier and $I_{d}$ is the set of indices of $N_{d}$ modulated sub-carriers, i.e., $I_{d} \subset\left\{-\frac{N}{2},-\frac{N}{2}+1, \ldots, \frac{N}{2}-1\right\}$. Further, as each sub-carrier transmits a symbol taken from an unit-energy $M_{I} \times M_{Q}$ rectangular constellation, which is formed by a sequence of $\left(m_{I}+m_{Q}\right)$ independent and equiprobable information bits, the BER of the $n^{\text {th }}$ sub-carrier can be decomposed as:

$$
B E R_{n}=\frac{1}{m_{I}+m_{Q}} \sum_{u, \beta} B E R_{n, u}^{\beta}\left(M_{\beta}, d_{\beta}\right),
$$




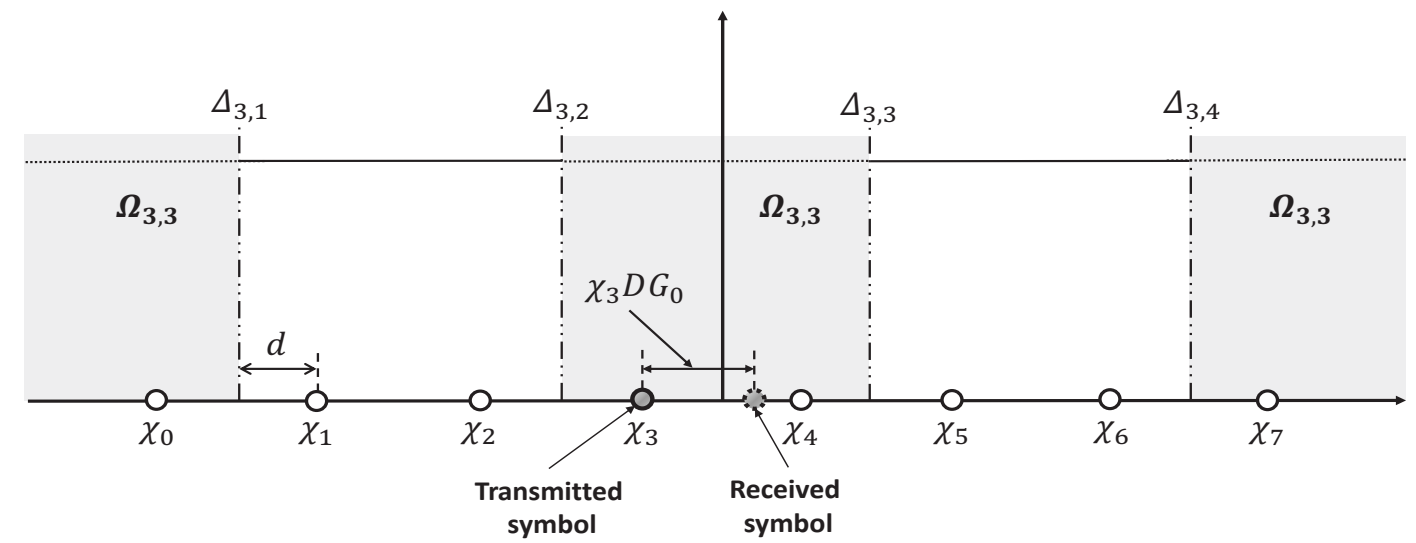

Fig. 3. An illustration of the decision regions and boundaries required to derive the $B E R_{n, u, v}^{\beta}(M, d)$ expression for $M=8, u=3, v=3$.

where $\beta \in\{I, Q\}$ refers to the in-phase and quadrature dimensions of the signal, $m_{\beta}=\log _{2} M_{\beta}, u \in\left\{1,2, \ldots, m_{\beta}\right\}$, and $d_{\beta}$ denotes the half minimum Euclidean distance in the $\beta$-dimension [13]. In (6), $B E R_{n, u}^{\beta}\left(M_{\beta}, d_{\beta}\right)$ is the BER corresponding to the transmitted information bit $b_{u}$ which is the $u^{\text {th }}$ bit in the $\beta$-dimension carried on the $n^{\text {th }}$ subcarrier. The demodulation of the received QAM signal is achieved by performing two quadrature PAM demodulations. Let us denote the $\beta$-dimension PAM constellation points as $\chi_{v}(M, d)$, where $v \in\{0,1, \ldots, M-1\}, M=M_{\beta}$ and $d=d_{\beta}$. We then have:

$$
\chi_{v}(M, d)=(2 v+1-M) d \triangleq S_{v}(M) d .
$$

Further, we denote the $\log _{2} M$ bit sequence that corresponds to the constellation point $\chi_{v}(M, d)$ as $\hat{\mathbf{b}}^{(v)}=$ $\left(\hat{b}_{1}^{(v)}, \hat{b}_{2}^{(v)}, \ldots, \hat{b}_{\log _{2} M}^{(v)}\right)$ :

$$
\chi_{v}(M, d) \leftrightarrow \hat{\mathbf{b}}^{(v)} .
$$

Assuming the $M$ bit sequences $\hat{\mathbf{b}}^{(v)}$ are equally probable, $B E R_{n, u}^{\beta}(M, d)$ in (6) can be calculated by averaging over all constellation points $\chi_{v}(M, d)$, resulting in:

$$
B E R_{n, u}^{\beta}(M, d)=\frac{1}{M} \sum_{v=0}^{M-1} B E R_{n, u, v}^{\beta}(M, d) .
$$

Taking into account (3), $B E R_{n, u, v}^{\beta}(M, d)$ corresponds to:

$$
\begin{array}{r}
B E R_{n, u, v}^{\beta}(M, d)=\operatorname{Pr}\left[\left(\chi_{v}(M, d)\right.\right. \\
\left.\left(1+D G_{0}\right)+\frac{1}{\sqrt{E_{s}}} \Phi_{n}\right)^{(\beta)} \\
\left.\notin \Omega_{u, v}(M, d) \mid \chi_{v}(M, d)\right],
\end{array}
$$

where $(z)^{(\beta)}$ is defined as:

$$
(z)^{(\beta)}=\left\{\begin{array}{ll}
\Re\{z\} & , \text { if } \beta=I \\
\Im\{z\} & , \text { if } \beta=Q
\end{array},\right.
$$

and $\Re\{z\}$ and $\Im\{z\}$ denote the real and imaginary part of $z$, respectively. Further, in (10), $\Omega_{u, v}(M, d)$ indicates the interval of $\frac{1}{\sqrt{E_{s}}}\left(R_{n}\right)^{(\beta)}$ from (3) for which the $u^{t h}$ bit $b_{u}$ is decided to equal the value of the $u^{t h}$ bit $\hat{b}_{u}^{(v)}$ corresponding to the $v^{t h}$ constellation point (see (8)). Finally, $\Phi_{n}$ is the interference plus noise, given by:

$\Phi_{n}=\sqrt{E_{s}} \sum_{i=-\frac{L}{2}, i \neq 0}^{\frac{L}{2}-1} D G_{i} X_{n-i \frac{N}{L}}+\sum_{i=-\frac{L}{2}}^{\frac{L}{2}-1} D G_{i} W_{n-i \frac{N}{L}}+W_{n}$

To further simplify (10), we exploit the fact that the mapping from (8) is a BRGC mapping [10]. In this case, the bit sequence $\left(\hat{b}_{1}^{(v)}, \hat{b}_{2}^{(v)}, \ldots, \hat{b}_{\log _{2} M}^{(v)}\right)$ equals $(v)_{2} \oplus\left(\left\lfloor\frac{v}{2}\right\rfloor\right)_{2}$, where $(v)_{2}$ denotes the natural binary code of integer $v$, and $\lfloor v\rfloor$ denotes the largest integer smaller than $v$. Moreover, for the BRGC mapping rule, the boundaries of the interval $\Omega_{u, v}(M, d)$ in (10) can be expressed as [12] (see an example in Fig. 3):

$$
\Delta_{u, y}(M, d) \triangleq B_{u, y}(M) d
$$

where $u \in\left\{1,2, \ldots, \log _{2} M\right\}, y \in\left\{1,2, \ldots, 2^{u-1}\right\}$, and $B_{u, y}(M)$ is given by:

$$
B_{u, y}(M)=(2 y-1) \cdot 2^{\log _{2} M-u+1}-M .
$$

The interference plus noise term $\Phi_{n}$ from (12) consists of a sum of random variables, i.e., $W_{n}$ and $D G_{i} W_{n-i \frac{N}{L}}$, which are Gaussian distributed as the gain mismatch contribution $D G_{i}$ can be considered constant, and $D G_{i} X_{n-i \frac{N}{L}}$, which depends on the interfering data symbols. When the number $L$ of sub-ADCs is sufficiently large, we can use the central limit theorem to approximate the sum over the interfering data symbols as a Gaussian random value. Hence, we model the total noise $\Phi_{n}$ as a Gaussian random variable with zero mean and variance $\sigma_{\beta}^{2}$ in the $\beta$-dimension (see appendix A). Note that $\sigma_{\beta}^{2}$ does not depend on the sub-carrier index $n$. As also the data contribution in (3) $\sqrt{E_{s}}\left(1+D G_{0}\right) X_{n}$ is independent of the sub-carrier index, because we assume the data symbols transmitted on the different sub-carriers belong to the same constellation and are equally probable, $B E R_{n}$ (5) becomes independent of the sub-carrier index. In the following, we drop the sub-carrier index $n$ for notational convenience. It follows that $B E R_{n, u, v}^{\beta}(M, d)=B E R_{u, v}^{\beta}(M, d)$ from (10) is of the 
following form:

$$
\begin{aligned}
& \operatorname{BER} R_{u, v}^{\beta}(M, d) \\
& =\frac{1}{2} \sum_{y=1}^{F_{u, v}(M)} \lambda_{u, v, y}(M) \operatorname{erfc}\left(\Gamma_{u, v, y}\left(M, d, \sigma_{\beta}^{2}\right)\right) \\
& +\frac{1}{2} \sum_{y=F_{u, v}(M)+1}^{2^{u-1}} \rho_{u, v, y}(M) \operatorname{erfc}\left(-\Gamma_{u, v, y}\left(M, d, \sigma_{\beta}^{2}\right)\right) .
\end{aligned}
$$

In (15), the BER consists of two sums, corresponding to the decision boundaries (for given $u$ ) at the left and right of the $v^{t h}$ constellation point, respectively. The number of terms in the sums depend on $F_{u, v}(M)$, which indicates the number of boundaries left of the considered constellation point. For BRGC:

$$
F_{u, v}(M)=\left\lfloor(2 v+1) 2^{-\left(\log _{2} M-u+2\right)}+2^{-1}\right\rfloor .
$$

Further, $\operatorname{erfc}($.$) is the complementary error function (erfc-$ function) defined by [14]: $\operatorname{erf} c(x)=\frac{2}{\sqrt{\pi}} \int_{x}^{\infty} e^{-z^{2}} d z$, and the argument $\Gamma_{u, v, y}\left(M, d, \sigma_{\beta}^{2}\right)$ of the erfc-function is defined as:

$$
\Gamma_{u, v, y}\left(M, d, \sigma_{\beta}^{2}\right)=\left(D_{u, v, y}(M)+S_{v}(M) D G_{0}\right) d \sqrt{\frac{E_{s}}{2 \sigma_{\beta}^{2}}},
$$

where $D_{u, v, y}(M)$ is a measure for the location of the $v^{t h}$ constellation point with respect to the $y^{t h}$ bound (for given $u$ ), and is given by:

$$
D_{u, v, y}(M)=S_{v}(M)-B_{u, y}(M),
$$

with $S_{v}(M)$ from (7) and $B_{u, y}(M)$ from (14). Finally, the pre-factors $\lambda_{u, v, y}(M)$ and $\rho_{u, v, y}(M)$ in (15) take the values +1 or -1 according to:

$$
\lambda_{u, v, y}(M)=(-1)^{\left\lfloor 2^{u-2-\log _{2} M}\left(D_{u, v, y}(M)-1\right)\right\rfloor}
$$

and

$$
\rho_{u, v, y}(M)=(-1)^{\left\lfloor 2^{u-2-\log _{2} M}\left(-D_{u, v, y}(M)-1\right)\right\rfloor} .
$$

Because of the symmetry of the constellation points and the BRGC mapping rule, it is easily verified that, for a given $u$, each set $(v, y)$, with $v \in\{0,1, \ldots, M-1\}$ and $y \in\left\{1,2, \ldots, F_{u, v}(M)\right\}$, corresponds to a unique set $(\tilde{v}, \tilde{y})$, with $\tilde{v} \in\{0,1, \ldots, M-1\}$ and $\tilde{y} \in$ $\left\{F_{u, \tilde{v}}(M)+1, F_{u, \tilde{v}}(M)+2, \ldots, 2^{u-1}\right\}$, so that: $S_{v}(M)=$ $-S_{\tilde{v}}(M)$ and $B_{u, y}(M)=-B_{u, \tilde{y}}(M)$, yielding: $\lambda_{u, v, y}(M)$ $=\rho_{u, \tilde{v}, \tilde{y}}(M)$ and $D_{u, v, y}(M)=-D_{u, \tilde{v}, \tilde{y}}(M)$. This results in:

$$
\begin{aligned}
& \sum_{v=0}^{M-1} \operatorname{BER} R_{u, v}^{\beta}(M, d) \\
& =\sum_{v=0}^{M-1} \sum_{y=1}^{F_{u, v}(M)} \lambda_{u, v, y}(M) \operatorname{erfc}\left(\Gamma_{u, v, y}\left(M, d, \sigma_{\beta}^{2}\right)\right) .
\end{aligned}
$$

Substituting (6), (9) and (15) into (5), and taking into account (21) and the independence of $B E R_{n, u, v}^{\beta}(M, d)=$ $B E R_{u, v}^{\beta}(M, d)$ on $n$, the BER for an unit-energy $M_{I} \times M_{Q}$
QAM constellation impaired by a gain mismatch of a TI-ADC realization is given by:

$$
\begin{aligned}
& B E R=\frac{1}{\left(m_{I}+m_{Q}\right)} . \\
& \sum_{u, \beta, v, y} \frac{\lambda_{u, v, y}\left(M_{\beta}\right)}{M_{\beta}} \operatorname{erfc}\left(\Gamma_{u, v, y}\left(M_{\beta}, d_{\beta}, \sigma_{\beta}^{2}\right)\right),
\end{aligned}
$$

where $\beta \in\{I, Q\}, m_{\beta}=\log _{2} M_{\beta}, u \in\left\{1,2, \ldots, \log _{2} M_{\beta}\right\}$, $v \in\left\{0,1, \ldots, M_{\beta}-1\right\}, y \in\left\{1,2, \ldots, F_{u, v}\left(M_{\beta}\right)\right\}$, and $\Gamma_{u, v, y}\left(M_{\beta}, d_{\beta}, \sigma_{\beta}^{2}\right), F_{u, v}\left(M_{\beta}\right)$ and $\lambda_{u, v, y}\left(M_{\beta}\right)$ are defined in (17), (16) and (19), respectively. The corresponding BER expression for an $M_{s}$-ary square QAM constellation is obtained by setting $M_{I}=M_{Q}=\sqrt{M_{s}}$ and $d_{I}=d_{Q}$. Similarly, the BER expression for an $M_{p}$-ary PAM constellation is derived by setting $M_{I}=M_{p}, M_{Q}=1$ and the sum over the $\mathrm{Q}$ dimension disappears. The expression (22) provides an efficient and fast approach to evaluate the BER performance compared to Monte-Carlo computation which can be very time consuming.

\section{NUMERICAL RESULTS}

In order to evaluate the accuracy of the derived BER expression, we compare the analytical results with MonteCarlo simulation results for various constellations and different mismatch levels. We assume $N=2048$. We generate $L$ independent gain mismatch values $d g_{l}^{(100 \%)}$ according to a uniform distribution over the interval $[-1,1]$ and keep these fixed. These $L$ values form the basis for all shown simulations. They can be interpreted as $100 \%$ level gain mismatch values corresponding to a particular TI-ADC implementation. The level of mismatch will be varied by scaling the $d g_{l}^{(100 \%)}$ gain mismatch values, i.e., for an $x \%$ mismatch level, we use as the gain mismatch values: $d g_{l}^{(x \%)}=\frac{x}{100} d g_{l}^{(100 \%)}$.

In Fig. 4, the BER performance for a system impaired by a gain mismatch level of $5 \%$ or $10 \%$ is compared to the BER performance of a system without gain mismatch. Results are presented for rectangular QAM $\left(\frac{d_{Q}}{d_{I}}=2\right)$, square QAM $\left(\frac{d_{Q}}{d_{I}}=1\right)$ and PAM constellations with different modulation orders when the number $L$ of sub-ADCs equals 256 (note that in practice, the number $L$ of sub-ADCs can be larger. For example, $56 \mathrm{GSa} / \mathrm{s}$ 8-bit TI-ADC in $65 \mathrm{~nm}$ CMOS technology, designed by Fujistu for 100 Gbps communication systems, interleaves up to 320 successive approximation register ADCs [15].). It can be seen that the theoretical BER curves correspond well with the simulation results. Further, Fig. 4 illustrates that the BER performance degradation becomes more severe when the mismatch level increases.

Fig. 5 shows the empirical and theoretical BER results for 16 QAM and $20 \%$ mismatch when the number $L$ of sub-ADCs is $4,8,16$ and 32 . It can be observed that the analytical BER curves are not in agreement with the simulated BER curves when $L$ equals 4,8 and 16 . In fact, the simulated performance is significantly better than that predicted by the theoretical expressions. This implies that the derived BER expression overestimates the effect of the gain mismatch. The deviation between theory and simulation decreases with increasing $L$. When $L$ equals 32 , the theoretical results match with the simulations. The deviation between the theoretical results and 
(a)

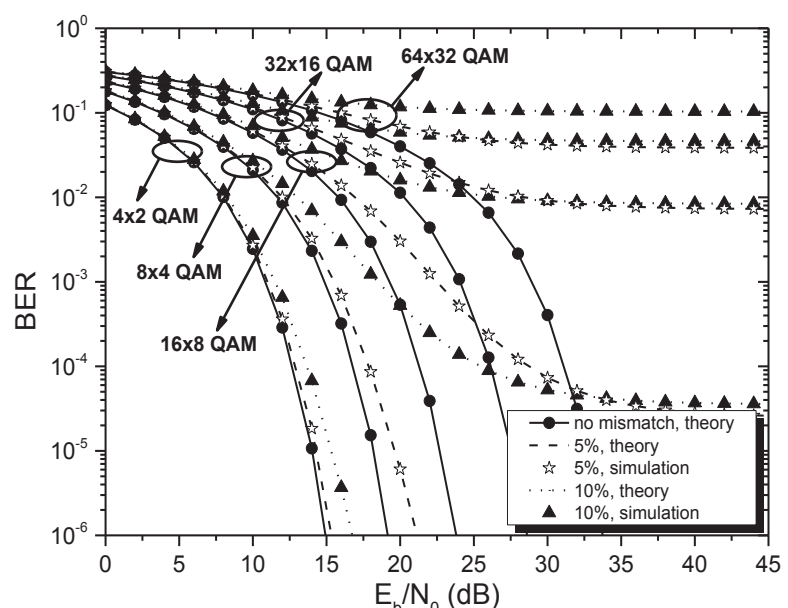

(b)

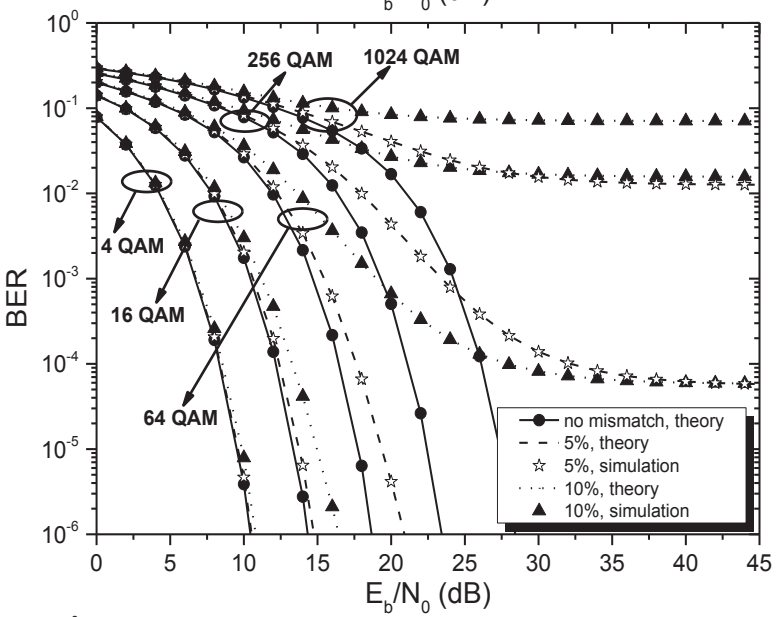

(c)

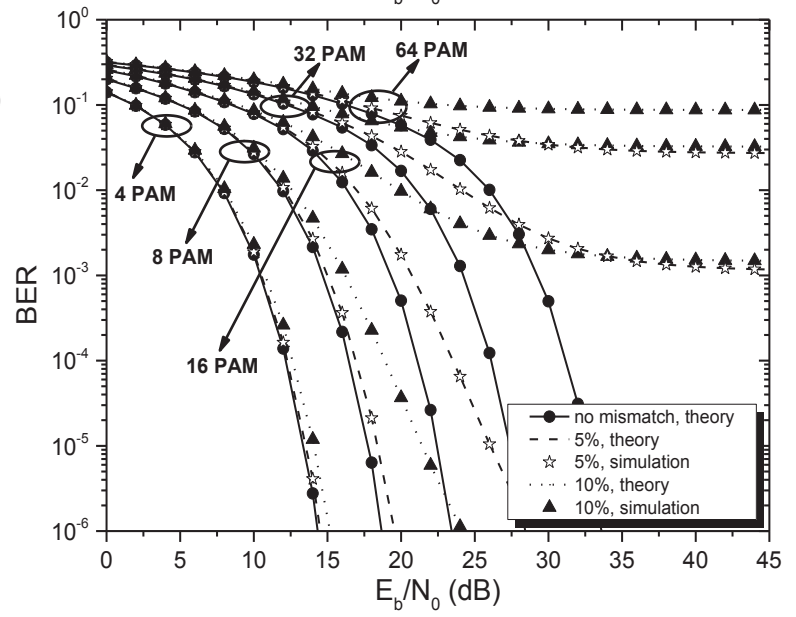

Fig. 4. BER curves for 256 sub-ADCs and different mismatch levels: (a) rectangular QAM $\left(\frac{d_{Q}}{d_{I}}=2\right)$, (b) square QAM $\left(\frac{d_{Q}}{d_{I}}=1\right)$, (c) PAM.

the simulations can be explained by the fact that when $L$ is small, $\Phi_{n}$ in (12) can no longer be approximated as a complex Gaussian random variable because the central limit theorem does not hold. Although the proposed theoretical expression for the BER does not hold for small values of $L$, it can still serve as an upper bound on the BER. Taking into account that in the coming years, the number of sub-ADCs will rapidly increase to achieve an even higher sampling rate, the approximate BER expression presented in this paper are

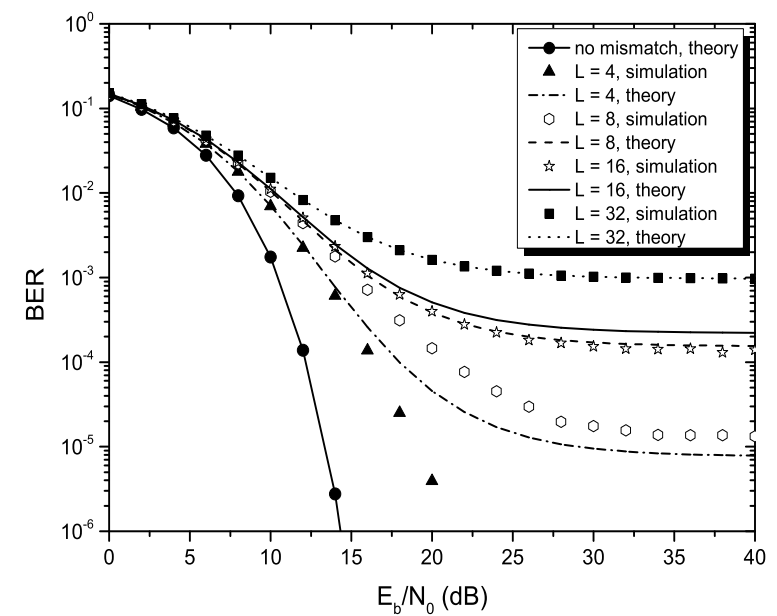

Fig. 5. BER curves for 16 QAM and $20 \%$ mismatch when $L$ equals 4,8 , 16 and 32 .

expected to be a useful tool to evaluate the performance of future TI-ADCs.

\section{CONCLUSIONS}

In this paper, we used a Gaussian approximation approach to derive an approximate closed-form BER expression for PAMand QAM-OFDM systems impaired by a gain mismatch of a TI-ADC realization, assuming AWGN channel and BRGC mapping. The proposed BER expression is very accurate for large $L$, and for a small number $L$ of sub-ADCs, it can serve as an upper bound on the BER performance.

\section{APPENDIX A \\ THE VARIANCE $\sigma_{\beta}^{2}$}

Let us decompose the total noise $\Phi_{n}$ in (12) as:

$$
\Phi_{n}=\Phi_{1, n}+\Phi_{2, n}+\Phi_{3, n}
$$

where $\Phi_{1, n}=\sqrt{E_{s}} \sum_{i=-\frac{L}{2}, i \neq 0}^{\frac{L}{2}-1} D G_{i} X_{n-i \frac{N}{L}}, \quad \Phi_{2, n}=$ $\sum_{i=-\frac{L}{2}, i \neq 0}^{\frac{L}{2}-1} D G_{i} W_{n-i \frac{N}{L}}$ and $\Phi_{3, n}=\left(1+D G_{0}\right) W_{n}$, respectively. It immediately follows that:

1) $\Phi_{1, n}, \Phi_{2, n}$ and $\Phi_{3, n}$ are statistically independent with zero mean.

2) $\Phi_{1, n}$ is the sum of i.i.d. random variables. Therefore, according to the central limit theorem, when the number $L$ of sub-ADCs is sufficiently large, $\Phi_{1, n}$ can be considered as a Gaussian distributed variable with variance 
$\sigma_{\Phi_{1}^{\beta}}^{2}$ per $\beta$-dimension, with

$$
\sigma_{\Phi_{1}^{\beta}}^{2}=\left\{\begin{array}{c}
E_{s} \xi \sum_{i=-\frac{L}{2}, i \neq 0}^{\frac{L}{2}-1}\left(\Re\left\{D G_{i}\right\}\right)^{2} \\
+E_{s}(1-\xi) \sum_{i=-\frac{L}{2}, i \neq 0}^{\frac{L}{2}-1}\left(\Im\left\{D G_{i}\right\}\right)^{2}, \text { if } \beta=I \\
E_{s} \xi \sum_{i=-\frac{L}{2}, i \neq 0}^{\frac{L}{2}-1}\left(\Im\left\{D G_{i}\right\}\right)^{2} \\
+E_{s}(1-\xi) \sum_{i=-\frac{L}{2}, i \neq 0}^{\frac{L}{2}-1}\left(\Re\left\{D G_{i}\right\}\right)^{2}, \text { if } \beta=Q
\end{array}\right.
$$

where $\xi$ is the average energy per symbol in the I dimension of the constellation (note that since the constellation was considered to have unit-energy, the average energy per symbol in the $\mathrm{Q}$ dimension of the constellation is $1-\xi$.).

3) $\Phi_{2, n}$ and $\Phi_{3, n}$ are Gaussian distributed random variables with respective variances per I/Q dimension :

$$
\sigma_{\Phi_{2}^{\beta}}^{2}=\frac{N_{0}}{2} \sum_{i=-\frac{L}{2}, i \neq 0}^{\frac{L}{2}-1}\left|D G_{i}\right|^{2},
$$

and

$$
\sigma_{\Phi_{3}^{\beta}}^{2}=\frac{N_{0}}{2}\left(1+D G_{0}\right)^{2} .
$$

In conclusion, for larger $L$, the quantity $\Phi_{n}$ from (12) and (23) can be considered as a complex-valued Gaussian random variable with independent real and imaginary components, having zero mean and variance $\sigma_{\beta}^{2}$ in the $\beta$-dimension given by:

$$
\sigma_{\beta}^{2}=\sigma_{\Phi_{1}^{\beta}}^{2}+\sigma_{\Phi_{2}^{\beta}}^{2}+\sigma_{\Phi_{3}^{\beta}}^{2},
$$

where $\sigma_{\Phi_{1}^{\beta}}^{2}, \sigma_{\Phi_{2}^{\beta}}^{2}$, and $\sigma_{\Phi_{3}^{\beta}}^{2}$ are defined as in (24), (25) and (26), respectively.

\section{ACKNOWLEDGMENT}

This research has been funded by the Interuniversity Attraction Poles Programme initiated by the Belgian Science Policy Office. The authors acknowledge the financial support from the Flemish fund for Scientific Research (FWO).

\section{REFERENCES}

[1] R. V. Nee and R. Prasad, OFDM for Wireless Multimedia Communications, Boston, MA: Artech House, 2000.

[2] J. Armstrong, "OFDM for Optical Communications," J. Lightwave Technol., vol. 27, no. 1, pp. 189-204, Feb. 2009.

[3] W. C. Black and D. A. Hodges, "Time Interleaved Converter Arrays," IEEE J. Solid-State Circuits, vol. SSC-15, no. 6, pp. 1022-1029, Dec. 1980.

[4] V-T-D. Huynh, N. Noels, P. Rombouts, J. Armstrong, and H. Steendam, "Effect of Time-Interleaved Analog-to-Digital Converter Mismatches on OFDM Performance," IEEE Inter. OFDM Workshop, pp. 128-135, Aug. 2014.

[5] S. W. Sin, S. P. U and R. P. Martins, Generalized Low-Voltage Circuit Techniques for Very High-Speed Time-Interleaved Analog-to-Digital Converters, Springer, 2011.

[6] A. Petraglia and S. K. Mitra, "Analysis of Mismatch Effects Among A/D Converters in a Time-Interleaved Waveform," IEEE Trans. Instrum. Meas., vol. 37, no. 5, pp. 831-835, Oct. 1991 .

)

[7] J. Elbornsson, F. Gustafsson, and J-E. Eklund, "Analysis of Mismatch Effects in a Randomly Interleaved A/D Converter System," IEEE Trans. on Circuit and Systems, vol. 52, no. 3, 2005.

[8] C. Vogel, "The Impact of Combined Channel Mismatch Effects in TimeInterleaved ADCs," IEEE Trans. Instrum. Meas., vol. 54, no. 1, pp. 415427, Feb. 2005.

[9] S. Ponnuru, M. Seo, U. Madhow and M. Rodwell, "Joint Mismatch and Channel Compensation for High-Speed OFDM Receivers with TimeInterleaved ADCs," IEEE Trans. Coтmu., vol. 58, no. 8, pp. 2391-2401, Aug. 2010.

[10] M. Gardner, Knotted Doughnuts and Other Mathematical Entertainments, W.H. Freeman \& Company, 1986.

[11] H. Johansson and P. Lowenborg, "Reconstruction of Non-Uniformly Sampled Bandlimited Signals by Means of Digital fractional Delay Filters," IEEE Trans. Signal Process., vol. 50, no. 11, pp. 2757-2767, Nov. 2002.

[12] P. K. Vitthaladevuni and M. S. Alouini, "A Closed-Form Expression for The Exact BER of Generalized PAM and QAM Constellations," IEEE Trans. Comm., vol. 52, no. 5, pp. 698-700, May 2004.

[13] K. Cho, and D. Yoon, "On the General BER Expression of One- and Two-Dimensional Amplitude Modulations," IEEE Trans. Comm., vol. 50, no. 7, pp. 1074-1080, Jul. 2002.

[14] W. T. Webb and L. Hanzo, Modern Quadrature Amplitude Modulation, IEEE Press, 1994

[15] P. Bower, I. Dedic, "High speed converters and DSP for 100G and beyond," Opt. Fiber Technol., Elsevier 2011. 